\title{
Practical Domain
}

National Cancer Institute

\section{Source}

National Cancer Institute. Practical Domain. NCl Thesaurus. Code C121208.

A domain of the General Adaptive Composite Score that is made up of scores from selfcare, home/school living, community use, and health and safety skills assessments. 